\title{
Fluorescence kinetics of tryptophan in a heterogeneous environment.
}

Olaf J. Rolinski ${ }^{{ }^{*}}$ and Vladislav Vyshemirsky ${ }^{2}$

${ }^{1}$ Photophysics Group, Centre for Molecular Nanometrology, Department of Physics, Scottish Universities Physics Alliance, University of Strathclyde, 107 Rottenrow, Glasgow G4 0NG, UK.

${ }^{2}$ School of Mathematics and Statistics, University of Glasgow, Glasgow G12 8QQ, UK.

Keywords: protein fluorescence, rotamer model, dielectric relaxation model, non-Debye kinetics, human serum albumin.

\begin{abstract}
The potentially highly informative, but complex fluorescence decay of amino acids in protein is not fully understood and presents a barrier to understanding. Here we have tested a new and general approach to describing experimentally measured the fluorescence decay in a heterogeneous macroscopic sample. The decay parameters carry information on the features of the kinetics induced by the environment's heterogeneity. Bayesian interference demonstrated that the model fits well to the fluorescence decay of tryptophan in Human Serum Albumin (HSA). The approach has the potential to accelerate photophysical research of heterogeneous media and, specifically, to solve a critical outstanding problem in interpreting protein fluorescence, paving the way to further progress in biomedical research.
\end{abstract}




\section{Introduction}

The intrinsic fluorescence of a protein carries information on its conformation and activity. However, the complex fluorescence decay of intrinsic fluorophores in protein, namely phenylalanine, tyrosine and tryptophan, is still not fully understood. Interpretation of fluorescence the decay exhibited by fluorescent amino acids in biological systems is a part of a bigger, unresolved, problem of explaining the multifarious decay observed in heterogeneous media, traditionally analysed by fitting to multi-exponential functions. After several decades of research into protein fluorescence decay using such analysis, two dominating models have emerged: the rotamer model and the model of dielectric relaxation.

The rotamer model [1-5] states that the discrete ground-state conformations of fluorophores (rotamers) exhibit different decay times, as each rotamer has different paths and rates of depopulation of the excited state. The model attributes the components of multi-exponential decays, or the peaks in the lifetime distributions, to individual rotamers.

In the relaxation model [6,7], the fluorophores are in a single conformation, but their fluorescence spectrum shift to longer wavelengths on a nanosecond time scale due to a solvent response to the excitedstate dipole. The multi-exponentiality of the observed decay is explained by the loss of fluorescence intensity, and thus adding a short-lifetime component, at short wavelengths. Conversely, the increase in fluorescence intensity at long wavelengths adds an increasing component (with negative amplitude) at long wavelengths of the spectrum. The main experimental argument supporting the relaxation model is the lifetime-wavelength correlation, that is, the mean decay lifetimes increasing with the detection wavelengths.

Whilst the lack of explanation for lifetime-wavelength correlation in the rotamer model is used as evidence against it; the lack of the common appearance of negative amplitudes in protein decays questions the validity of the relaxation model. This ambiguity surrounding the interpretation of protein autofluorescence has hampered the development of protein fluorescence lifetime-based research and its application in biomedical sciences. Some progress has been achieved recently by quantum mechanics molecular mechanics simulations [8], which explain the lifetime-wavelength correlation within the rotamer model, suggesting that rotamers emitting at shorter wavelengths are more likely to be involved in charge transfer, and thus they have shorter lifetimes.

The purpose of this paper is to apply a statistical approach which does not rule out multiple conformations and dielectric relaxation, but, instead of considering interactions of individual fluorophores, calculates fluorescence response of a macroscopic sample, which results in other than multi-exponential 
fluorescence decay. Briefly, the heterogeneous environment is modelled by a completely assymetric $\alpha$ stable distribution of the transition rates $k$ of depopulation of the excited state, with an impact on the fluorescence decay of the macroscopic sample. Validity of this model is demonstrated on the example of tryptophan in human serum albumin (HSA) and its applicability to the whole area of fluorescence in heterogeneous media is discussed.

\section{Kinetic model}

Here, we assume that the probability of a randomly selected fluorophore to remain in the excited state at time $t$ after excitation decays in two stages. In the first stage, from time 0 up to the certain time $t_{c}$ called maximum quenching time, the decay is exponential with the rate $k=k_{r}+k_{n r}+k_{q}$, which is the sum of the radiative, nonradiative and quenching transition rates, respectively. In this stage, the fluorophore is likely to be quenched, e.g. due to charge transfer or other environment-induced non-radiative transition, thus $k$ is relatively high. If, however, the fluorophore survived in the excited state by the time $t_{c}$, the probability of remaining in the excited state in the second stage, ie for $t>t_{c}$, decreases substantially slower. In the approximation considered in this paper, it stops decaying and remains constant for the time exceeding the time range when the fluorescence is detected in lifetime experiment. As we show later, this is not in contradiction to the fluorescence always decaying to zero.

The radiative transition rate $k_{r}$ is the same for all fluorophores. Nonradiative $k_{n r}$ and quenching $k_{q}$ transition rates, however, are not the same to all fluorophores, due to assumed heterogeneity of the molecular system. This leads to the decay rate density function $f_{i}(k)=d F_{i}(k) / d k$, where $F_{i}(k)$ is the probability that the decay rate of the $i$-th fluorophore is less or equal to $k$. Again, due to heterogeneity of the environment, the value of $t_{c}$ is not the same for each fluorophore, but is characterised by the density distribution function $h\left(t_{c}\right)$.

Signal detected in a fluorescence lifetime experiment is a statistical representation of the photons emitted by a large number $N \rightarrow \infty$ of fluorophores, each at an individual state of interaction with its surrounding at the moment of fluorescence transition. Let $t_{i}$ be the moment in time when the $i$-th fluorophore loses its excitation (due to a radiative or non-radiative transition). If the transient rate of decay of the $i$-th fluorophore $b_{i}=k>0$, and $d_{i}=t_{c}$, the conditional probability that the fluorophore is still in the excited state at time $t$ is

$$
P\left(t_{i} \geq t \mid b_{i}=k, d_{i}=t_{c}\right)=\exp \left[-k \min \left(t, t_{c}\right)\right]
$$


The photophysics of individual molecule expressed in the eqtn (1) can be now used to obtain the expression for the macroscopically observable fluorescence decay $I(t)$. The formula (1) has been previously proposed to describe dipole kinetics by Weron [9,10] and Weron and Jurlewicz [11], for dielectric relaxation phenomena in condensed systems. Here we discuss the key points of this model in the context of its applicability to fluorescence kinetics and adopt the derivation from the Ref. 9-11 to obtain the expression for $I(t)$. No particular mechanisms resulting in the specific density distributions $f(k)$ and $h(t)$ are assumed. Instead, we search for such functions, for which $I(t)$ exists and is a positive function decreasing to zero.

In the system of $N$ fluorophores of the decay rate values $k$ characterised by the density distribution $f(k)$ and the maximal quenching times $t_{c}$ by the density distribution $h\left(t_{c}\right)$, the time derivative of the probability $P\left(t_{i, N} \geq t / A_{N}\right)$, that the randomly selected i-th molecule is still in the excited state at time $t$, is given by [11]

$$
\frac{d}{d t} P\left(t_{i, N} \geq \frac{t}{A_{N}}\right)=\left(1-H_{N}\left(\frac{t}{A_{N}}\right)\right) \frac{d}{d t} L\left(F ; \frac{t}{A_{N}}\right)
$$

where $A_{N}$ is a normalising constant, $h_{N}\left(t_{c}\right)=d H_{N}\left(t_{c}\right) / d t_{c}$ and

$$
\int_{0}^{\infty} f(k) \exp \left[-k \frac{t}{A_{N}}\right] d k=L\left(F ; \frac{t}{A_{N}}\right)
$$

is the Laplace transform of $f(k)$. Because the moments of transitions $t_{i, N}$ are independent and identically distributed, the probability, that all initially excited molecules are still in the excited state at time $t$ is

$\Phi(t)=\lim _{N \rightarrow \infty}\left[P\left(t_{i, N} \geq \frac{t}{A_{N}}\right)\right]^{N}$

$\Phi(t)$ can be regarded as proportional to the population of the excited fluorophores at time $t$ after excitation. However, the limiting value in the eqtn (4) exists only if $F(k)$ and $H(t)$ satisfy certain mathematical conditions. Weron and Jurlewicz demonstrated [11], that the $F(k)$ has to be a completely asymmetric $\alpha$-stable distribution belonging to the domain of attraction of Levy-stable law and $H(t)$ has to belong to the domain of attraction of the max-stable law of type II.

This finding is based on two theorems. Firstly, the $N$-th power of the Laplace transform for the nondegenerate distribution function $F(k)$ converges to the non-degenerate limiting transform as $N$ tends to infinity if, and only if, $F(k)$ belongs to the domain of attraction of the Lévy-stable law, i.e. if follows the condition 
$\lim _{b \rightarrow \infty} \frac{1-F(x b)}{1-F(b)}=x^{-\alpha}$

for some $\alpha, 0<\alpha<1$, called index of stability. If so, then for $A_{N}=N^{1 / \alpha}>0$

$\lim _{N \rightarrow \infty}\left[L\left(f ; t / N^{1 / \alpha}\right)\right]^{N}=\exp \left[-(A t)^{\alpha}\right]=\exp \left[-\left(\frac{t}{\tau_{0}}\right)^{\alpha}\right]$

where $A=1 / \tau_{0}$ is a positive constant. $\alpha$ depends on the detailed mechanisms of molecular interactions: for $\alpha \rightarrow 1$ the decay is exponential, $\alpha<1$ indicates that the distribution $f(k)$ is "heavy tailed" [9-11].

Secondly, as $N \rightarrow \infty, H_{N}\left(t / A_{N}\right)$ tends to a non-degenerate distribution function of non-negative random variable, only when $H_{N}\left(t / A_{N}\right)$ belongs to the domain of attraction of the max-stable law of type II [11], that is, it obeys the condition

$\lim _{t \rightarrow \infty} \frac{1-H_{N}(t x)}{1-H_{N}(t)}=x^{-\gamma}$

Ergo, for the normalising constant $A_{N} \sim N^{1 / \alpha}[11]$

$$
\lim _{N \rightarrow \infty} H_{N}\left(\frac{t}{N^{1 / \alpha}}\right)=\exp \left[-\frac{(A t)^{-\gamma}}{\kappa}\right]
$$

where $\gamma$ and $\kappa$ are positive constants, and $A$ is the same as in (6). Using (2) with (6)and (8) allows expressing $\Phi(\mathrm{t})$ in the form [11]:

$\Phi(t)=\exp \left[-\kappa^{-\alpha / \gamma} \int_{0}^{\alpha / \gamma}\left(t / \tau_{0}\right)^{\alpha}\left(1-\exp \left[-s^{-\gamma / \alpha}\right]\right) d s\right]$

where $\alpha$ is an index of stability of the stable distribution $f(k), \gamma$, and $\kappa$ are positive constants and $\tau_{0}$ is fluorescence lifetime. When $t$ tends to infinity, the $\Phi(t)$ tends to a positive value for $\alpha / \gamma<1$, equals 0 for $\alpha=\gamma$, and does not converge for $\alpha / \gamma>1$. As fluorophores cannot be in the excited state permanently, $\Phi(t)$, after long enough time, has to decay to zero. Therefore, we must choose the case $\alpha=\gamma$, obtaining

$$
\Phi_{\alpha, \kappa}(t)=\exp \left[-\frac{1}{\kappa} \int_{0}^{\kappa\left(t / \tau_{0}\right)^{\alpha}}(1-\exp [-1 / x]) d x\right]
$$

The relationship between the experimental fluorescence decay $I_{\alpha, k}(t)$ and the function $\Phi_{\alpha, k}(t)$, can be found from the formal kinetic equation for $\Phi_{\alpha, k}(t)$ 
$\frac{d \Phi_{\alpha, \kappa}(t)}{d t}=-k(t) \Phi_{\alpha, \kappa}(t)$

where $k(t)=k_{r}+k_{n r}(t)+k_{q}(t) . I_{\alpha, k}(t)$ can be defined as

$I_{\alpha, \kappa}(t)=\frac{k_{r}}{k(t)}\left(-\frac{d \Phi_{\alpha, \kappa}(t)}{d t}\right)$

which results in

$I_{\alpha, \kappa}(t)=k_{r} \Phi_{\alpha, \kappa}(t)=k_{r} \exp \left[-\frac{1}{\kappa} \int_{0}^{\kappa\left(t / \tau_{0}\right)^{\alpha}}(1-\exp [-1 / x]) d x\right]$

This formula is a fluorescence equivalent of the result obtained in [11]. As the generalisation of the exponential function, $I_{\alpha, k}(t)$ is more adequate to represent fluorescence decay in the heterogeneous environment. For the specific values of $\alpha$ and $\kappa$, it converts into simpler model decays, which are already known from fluorescence applications. The degenerate case $I_{1,0}(t) \sim \exp \left[-t / \tau_{0}\right]$ demonstrates that, when $\alpha=1$ and $\kappa=0$, the decay is single-exponential (see Fig.1a). In heterogeneous environment (resulting in $\alpha<1$ and $\kappa>0$ ), the reduced index of stability $\alpha$ modifies the whole decay, while the parameter $\kappa$ influences only the tail part. For $\kappa \rightarrow 0$, the decay function converges to $I_{\alpha, 0}(t) \sim \exp \left[-\left(t / \tau_{0}\right)^{\alpha}\right]$. These, so called stretched-exponentials (Fig.1b), have been discussed already in the context of fluorescence applications [14,15]. For $\alpha \rightarrow 1, I_{1, \kappa}(t) \sim\left(1+\kappa\left(t / \tau_{0}\right)\right)^{-1 / \kappa}$ (Fig.1c) becomes the Becquerel function [15,16], and has been previously used in protein research, e.g. to study the effect of temperature on tryptophan fluorescence in NATA and HSA[17].

To investigate whether the effects of $\alpha<1$ and $\kappa>0$ may result in a fluorescence decay like those observed for protein, time-correlated single-photon counting [17]-type synthetic decay data were generated for a set of parameters $\tau_{0}, \alpha$ and $\kappa$ and then analysed by means of the maximum entropy method (MEM) [18,19]. The technique recovers the lifetime distribution functions $g(\tau)$, which are related to the fluorescence decay $I(t)$ by

$I(t)=\int_{0}^{\infty} e^{-t / \tau} g(\tau) d \tau$

The method we applied [19], uses Shannon-Jaynes entropy defined as $S=\int(g(\tau)-m(\tau)-g(\tau) \log [g(\tau) / m(\tau)]) d \tau$, where $m(\tau)$ is the model lifetime distribution that represents the prior knowledge on the system. The procedure maximizes the functional $S-\eta \chi^{2}$, where $\chi^{2}$ is the mean square error between the model and the data and $\eta$ is the Langrange multiplier. This approach is 
not applicable for negative amplitudes, but is frequently used by researchers to represent complex fluorescence in protein. Note that the alternative approach allowing negative amplitudes was also presented [20]. The number of channels used in simulations was 4000, the time calibration was 0.0125 ns/channel, and the maximum number of counts in the peak channel was set at 10000. Fig. 2a shows the lifetime distributions for $\tau_{0}=2 \mathrm{~ns}$, and different $\alpha$ and $\kappa$. When $\alpha=1$ and $\kappa \rightarrow 0$ (see inset), the MEM produces $g(\tau) \sim \delta\left(\tau-\tau_{0}\right)$, thus the decay is single-exponential. However, for $\alpha=1$ and $\kappa=0.1$, the recovered distribution indicates a 2-exponential decay, which is characteristic for some simple protein systems, e.g. for tyrosine (Tyr) and NAYA [21]. For $\kappa$ still 0.1 and $\alpha$ decreasing from 1 to lower values, the resulting lifetime distribution gradually becomes broader and more structured, with the peaks and mean lifetime shifting towards longer values. Similarly, for $\tau_{0}=2 \mathrm{~ns}, \alpha=0.9$ and gradually increasing $\kappa$ (Fig. $2 \mathrm{~b}$ ), the dominating peak shifts towards shorter values and the profile becomes more structured, with the mean lifetime becoming longer. We conclude that the multi-peak distributions in Fig.2 are characteristic for protein fluorescence decay (e.g. Refs 7, 21), and would be traditionally interpreted in terms of several rotamers, with a physical meaning attributed to each peak. According to our description, however, the peaks in Fig. 2 have no physical meaning and only the parameters $\tau_{0}, \alpha$ and $\kappa$ carry information on the kinetics. The above result also suggests that the decay (13) can be misinterpreted in real lifetime experiments as a multi-exponential function.

\section{Experimental setup and data fitting}

To verify the validity of the model decay $I_{\alpha, k}(t)$ in a real protein system, the fluorescence decays of tryptophan (Trp) in HSA, were measured at different wavelengths of detection $\lambda$ and temperatures $T$, and the results were analysed by means of the dependence (13).

HSA was purchased from Sigma-Aldrich (Poole, UK) and used without further purification. The solution of $30 \mu \mathrm{M}$ in $0.01 \mathrm{M}$ phosphate buffer, $\mathrm{pH} 7.4$, was prepared on the day of measurements. Fluorescence decays were measured using the time-correlated single-photon counting technique, on an IBH Fluorocube fluorescence lifetime system (Horiba Jobin Yvon IBH Ltd., Glasgow, U.K.). The instrument has been equipped with an AlGaN version of the pulsed light emitting diode, working at $295 \mathrm{~nm}$, in order to excite tryptophan only, and a temperature-controlled sample holder, connected to a Neslab RTE-11 thermostat (Thermo Scientific, U.K.).

To achieve least squares fits, we performed minimum search using standard optimisation algorithms of the cost function: 
$L\left(\alpha, \kappa, \tau_{0}\right)=\frac{1}{N-\gamma} \sum_{i=1}^{N} \frac{\left(F_{i}-P_{i}\left(\alpha, \kappa, \tau_{0}\right)\right)^{2}}{F_{i}}$

where $N$ is the number of channels considered in the experiment, $\gamma$ is the number of equation parameters, $F_{i}$ is the experimental measurement for channel $i$, and $P_{i}\left(\alpha, \kappa, \tau_{0}\right)$ is the model prediction for channel $i$ :

$P_{i}\left(\alpha, \kappa, \tau_{0}\right)=B+A \cdot E(t+\Delta) * I_{\alpha, \kappa}(t)$

here $t$ is the time corresponding to a reading from channel $i, E(t)$ is the measured excitation profile, and $A, B, \Delta$ are scaling, background level, and signal delay technical parameters respectively. Fitting demonstrated consistence between (16) and the data - see Fig. 3 as an example.

We have also performed data analysis using the Bayesian inference methods for identifying plausible distributions of model parameters that explain our experimental observations [22].

Formally, Bayesian inference is statistical inference in which evidence or observations are used to update or to infer the probability of belief in a given statement. To perform such inference we need to define a way to express our initial beliefs and describe the process by which some evidence or observations can be used to update these beliefs. Applying Bayesian inference methods requires the formal representation of the available knowledge. This should include the statistical model for the problem, and a priori information about the model parameters, as we assume that the statistical model is parametric. Our initial beliefs (initial state of information) about the values of parameters of each available statistical model of the system are, most often, uncertain and therefore distributed according to some probability density function $p(\theta \mid M)$. This probability distribution is called "a priori distribution of model parameters".

When some new information D about the modelled phenomenon is acquired, we update our beliefs according to Bayes' theorem. The updated distribution of our beliefs is called "a posteriori distribution of model parameters". D can correspond to the data from a newly performed experiment, or new information published in a recent paper. Bayes' theorem defines how the posterior can be obtained from the prior, generally:

$p(\theta \mid M, D)=\frac{p(D \mid M, \theta) p(\theta \mid M)}{\int p(D \mid M, \theta) p(\theta \mid M) d \theta}$

Here the probability $p(D \mid M, \theta)$ to produce data $\mathrm{D}$ with model $\mathrm{M}$ given parameters $\theta$ is called “likelihood”[23,24]. 
In this paper we consider a model defined using a convolution of discrete data with an integral function. In cases such as this, it is not possible to perform inference analytically due to the complexity of the integrals involved, and we need some numerical methods to be able to evaluate the posteriors, such as the Monte Carlo methods [25,26]. We employed the Sequential Monte Carlo (SMC) sampler proposed by Del Moral et al. [27] to find parameter posteriors for our models. This sampler employs a strategy of sequential importance sampling starting with an easy to sample prior distribution and eventually converging to the desired posterior, through a sequence of artificial intermediate distributions [28].

The statistical model of fluorescence considered in this paper can be defined with the following equations:

$$
\begin{aligned}
& Y=F_{y}+\varepsilon+\xi \\
& \varepsilon \sim N\left(0, F_{y} I\right) \\
& \xi \sim N\left(0, \sigma^{2} I\right) \\
& F_{y}\left(\alpha, \kappa, \tau_{0}, A, B, \Delta, t\right)=B+A \cdot F_{c o n v}\left(\alpha, \kappa, \tau_{0}, t+\Delta\right) \\
& F_{c o n v}\left(\alpha, \kappa, \tau_{0}, t\right)=E(t) * I\left(\alpha, \kappa, \tau_{0}, t\right) \\
& I\left(\alpha, \kappa, \tau_{0}, t\right)=\exp \left[-\frac{1}{\kappa} \int_{0}^{\kappa\left(t / \tau_{0}\right)^{\alpha}}(1-\exp [-1 / x]) d x\right]
\end{aligned}
$$

where $E(t)$ is the measured excitation profile, $\varepsilon$ is an error associated with data dispersion, and it is normally distributed around the deterministic model predictions with variance equal to $F_{y} . \xi$ is another error term associated with model mismatch, it is parameterised with unknown variance $\sigma^{2}$ (standard deviation $\sigma$ ).

Background intensity parameter $B$ was estimated as an average of the observation noise in the initial part of the dataset, before the excitation pulse was emitted. Six other parameters were estimated using methods for Bayesian parameter inference: $\alpha, \kappa, \tau_{0}, A, \Delta, \sigma$.

The following priors were used for our six free model parameters, these priors correspond to our subjective belief about suitable parameter values before observing any experimental data: 
$p(\alpha)=\left\{\begin{array}{c}0, \text { when } \alpha<0 \text { or } \alpha>1 \\ e^{-2}+p_{\exp }(1-\alpha, 0.5), \text { when } \alpha \in[0,1]\end{array}\right.$

$p(\kappa)=\left\{\begin{array}{c}0, \text { when } \kappa<0 \text { or } \kappa>1 \\ e^{-4}+p_{\text {exp }}(\kappa, 0.25), \text { when } \kappa \in[0,1]\end{array}\right.$

$p\left(\tau_{0}\right)=p_{\text {unif }}\left(\tau_{0} ; 0.01,10\right)$

$p(A)=p_{\text {unif }}(A ; 0.00001,1)$

$p(\Delta)=p_{\exp }(\mid \Delta \| ; 1)$

$p(\sigma)=p_{\exp }(\sigma ; 1000)$

The parameters were considered independent a priori. Parameter posteriors for two experiments described in this paper were inferred using the Sequential Importance Sampling algorithm [27]. The likelihood of observing the experimental data from the $I_{\alpha, k}(t)$ model has been defined as

$P\left(F \mid \alpha, \kappa, \tau_{0}, \sigma\right)=\prod_{i=1}^{N} N\left(F_{i}-P_{i}\left(\alpha, \kappa, \tau_{0}\right) \mid F_{i}+\sigma^{2}\right)$

where $N\left(\cdot \mid F_{i}+\sigma^{2}\right)$ is the Gaussian probability density function with mean 0 and variance $F_{i}+\sigma^{2}$.

\section{Results and discussion}

\subsection{Least-squares and Bayesian inference.}

In the traditional least-squares analysis, fitting $I_{\alpha, \kappa}(t)$ revealed a good consistency with the data, with almost all $\chi^{2}$ values in the range of $1.00 \leq \chi^{2} \leq 1.10$ and randomly distributed residuals. The resulting parameters are shown in Table 1 and Table 2.

Using methods of Bayesian inference of the parameters provided similar to the least-squares approach solutions and their uncertainties. We have found that the $95 \%$ credibility intervals for a posteriori (after considering the data) distributions of plausible parameter values were remarkably tight, suggesting that the problem is well defined and the parameter identity is not compromised. Naturally, the resulting posterior distribution is defined with a sample in 6 dimensions, which is difficult to visualise. We therefore display only the marginal posterior distributions of single model parameters, where the rest of the dimensions of the samples are integrated out. These can be thought of as projections of the 
multidimensional posterior distribution on individual axes. The box plots for these marginal posteriors are depicted in Figures 4 and 5. Note, that parameters $A$ and $\Delta$ are technical parameters that correspond to a particular measurement setup and therefore there is little if any dependency between experimental conditions their posterior distributions. It is remarkable that the posterior distributions of the model mismatch error standard deviation $\sigma$ (as displayed in Figure 5) are very small. It suggests that the model predictions match the experimental data extremely well.

We compare model predictions to the all experimental data, with the examples shown on Fig.6. The black points in these plots correspond to actual fluorescence measurements, black lines are mean posterior model predictions, light grey area corresponds to the $50 \%$ credibility interval of model predictions, while the dark grey area corresponds to the $95 \%$ credibility interval of model predictions.

\subsection{Comparison of $\mathrm{I}_{\alpha, \mathrm{K}}(\mathrm{t})$ with 2- and 3-exponential models.}

The key point in our discussion is comparison of the goodness of fit criteria for $I_{\alpha, k}(t)$ and for the traditional 3-exponential function. It can be shown that both functions fit well to the experimental data, but we have significantly higher order of a posteriori uncertainty about the 3-exponential model due to its higher complexity (5 physical and 3 technical parameters), instead of $I_{\alpha, k}(t)$ (3 physical and 3 technical parameters). While this explains why the 3-exponential function is a good representation of $\operatorname{Tr} p$ decay, it may not be a proper model of its kinetics. Indeed, the Ockham's razor principle guides us to select the model decay $I_{\alpha, k}(t)$ as more appropriate, because it contains less parameters. This can be confirmed by using both Bayesian model comparison methods and a more traditional approach using the Akaike Information Criterion (AIC). AIC provides a means for statistical model selection that deals with the trade-off between the goodness of fit and the complexity of the model. The approach has been developed to avoid the problem of over-fitting - a ubiquitous problem in modelling physical processes when a more complex model will always provide a better fit to data purely because of its flexibility. AIC is widely accepted as a preferred model selection tool in comparison to a simple likelihood ratio test [29]. Once we have the results of the traditional $\chi^{2}$ fit to data, we can easily compute corresponding AIC scores as $\mathrm{AIC}=\chi^{2}+2 k$, where $k$ is a number of model parameters. The smaller value of the AIC indicates the preferred model. Naturally, a substantial improvement in $\chi^{2}$ fit is required to justify using an extra parameter in a model. In our case, AIC analysis clearly favours the newly proposed model as more adequate explanation of the observed decays, than the 3-exponential function. Also, the AIC values for the 2-exponential model, presented here as an alternative decay characterised by the same number of the parameters as the $I_{\alpha, \kappa}(t)$ function, show advantage of the new model. 
Further comparison of the models has been performed on the example of the data collected at $330 \mathrm{~nm}$ and temperature $294 \mathrm{~K}$. The newly proposed model explains this data well as shown in Fig. 7a, but the tripleexponential model also provides a reasonable data prediction as can be observed in Fig. 7b. Moreover, the 3-exponential model, similarly to the newly proposed model, has relatively small posterior values for the standard deviation of the model mismatch error, as can be seen in the last row in Fig.8, which suggests, that this model also explains well the experimental data. However, 3-exponential model is considerably more complex than the $I_{\alpha, k}(t)$. Compare the marginal posterior distributions in Fig. 8 to the marginal posterior distribution for the newly proposed model depicted in Fig.9. The new model posterior over only six parameters is therefore defined in six dimensions, while the triple exponential one is defined in eight dimensions. Given that the variances of marginal distributions in Figs. 8 and 9 are comparable in size, the measure for the posterior of the $I_{\alpha, k}(t)$ model will therefore be much smaller than the one for the tripleexponential model. This illustrated that we have significantly higher order of a posteriori uncertainty about the triple-exponential model due to its higher complexity.

\subsection{Implications for Trp photophysics}

Recovered parameters $\tau_{0}, \alpha$ and $\kappa$, presented in Fig.4, provide new view on the photophysics of $\operatorname{Tr} p$ in HSA. An increase in the lifetime $\tau_{0}$ observed for the longer detection wavelenghts $\lambda$, supports fluorophore-solvent relaxation, and is accompanied by an increase in $\alpha$, implying a less heavy tailed distribution $f(k)$, i.e. smaller spread of the transition rates $k$ in fluorophores of lower excitation energy. Parameter $\kappa>0$ indicates the presence of a long lifetime component in the decay, and its contribution also increases with $\lambda$. The above effects can be explained by fluorophores emitting at longer wavelengths being either less likely to be quenched (like in the rotamer model [8]), or fluorescing at the later stage of solute-solvent relaxation (like in the relaxation model). The measurements vs. $T$, have shown an expected decrease in lifetime $\tau_{0}$ as a result of increased collisions of $\operatorname{Tr} p$ with the surroundings, which is followed by a slow decrease in $\alpha$, showing that $f(k)$ becomes more heavy tailed, i.e. the range of the transition rates $k$ becomes broader at higher temperature. As the parameter $\kappa$ drops, the contribution of the long lifetime process decreases with temperature, also due to increased collisions.

The ability of the proposed approach to reveal changes in the rate density distributions is a clear advantage of the proposed method over the traditional multi-exponential analysis. However, the eqtn (13) itself does not constitute a detailed model of protein fluorescence kinetics. Instead, it specifies what information on the kinetics is included in fluorescence decay of the real sample. In this case, the dependence of the recovered parameters $\tau_{0}, \alpha$ and $\kappa$ on $\lambda$ in Fig.4 suggests that the measured system consists of a number of sub-populations of fluorophores characterised by $\lambda$-dependent stable distributions 
$f_{\lambda}(k)$ and $h_{\lambda}(t)$, which is consistent with both the existence of rotamers and their dielectric relaxation. To create an adequate model of HSA photophysics, we currently study the effects of different experimental factors on $\tau_{0}$ and $\alpha$ (e.g. quenching), and on $\kappa$ (compounds promoting delayed fluorescence).

More generally, fluorescence decay in heterogeneous medium does not carry full information on the underlying kinetics (ie $f_{p}(k)$ and $h_{p}(t)$, where $p$ is an independent parameter or parameters of the experiment), but only limited information $\left(\tau_{0}(p), \alpha(p)\right.$ and $\left.\kappa(p)\right)$. To verify validity of a specific model of the kinetics, the model-derived $\tau_{0}(p), \alpha(p)$ and $\kappa(p)$ dependencies need to be compared with the experimental $\tau_{0}, \alpha$ and $\kappa$ values, recovered from fitting the eqtn (13) to the experimental decays for different $p$. This will enable extracting information from the fluorescence decays in a way which considers its statistical and limited nature. In fact, this approach can be beneficial to the whole area of fluorescence sensing.

\section{Conclusions}

Our method of describing fluorescence decay has demonstrated that simple molecular-level photophysics in heterogeneous environment implies non-exponential fluorescence decay, eqtn (13), of a real sample. The derived decay $I_{\alpha, k}(t)$ is parameterised by the fluorophore lifetime $\tau_{0}$ and index of stability $\alpha$, both reflecting the rate density distribution $f(k)$, and $\kappa$, which characterises the maximal quenching time density distribution function $h(t) . I_{\alpha, K}(t)$ is a statistically justified alternative for the dogmatic use of multiexponential functions. Many fluorophores rejected thus far as fluorescence lifetime sensors due to their "complex" kinetics, may follow the $I_{\alpha, k}(t)$ behaviour, thus demonstrating their sensing potential, with $\alpha$ and $\kappa$ as the reporting parameters.

In context of protein research, presented analysis shows that eqtn (13) sufficiently represents $\operatorname{Trp}$ fluorescence decay in HSA. We have also observed, that this formula fits well to fluorescence decays of other protein, simple peptides and some fluorophores (with $\kappa>0$ in almost all cases). This finding, combined with fluorescence decay modelling, can impact on our understanding of the numerous biomolecular systems, where the expected progress has not been achieved, because traditional descriptions were not fully conclusive.

\section{References:}


[1] Donzel B, Gaudcho P and Wahl P 1974 Study of conformation in excited state of 2 tryptohanyl diketopiperazines J.Am.Chem.Soc. 96(3) 801-808

[2] Szabo A G and Rayner D M 1980 Fluorescence decay of tryptophan conformers in aqueous J.Am.Chem.Soc. 102(2) 554-563

[3] Engh R A, Chen L X Q and Fleming G R 1986 Conformational dynamics of tryptophan - a proposal for the origin of the nonexponential fluorescence decay Chem.Phys.Letts. 126(3-4) 365-371

[4] Gordon H L, Jarrell H C, Szabo A G, et.al. 1992 Molecular dynamics simulations of the conformational dynamics of tryptophan J.Phys.Chem. 96(4) 1915-1921

[5] Dahms T E S, Willis K J and Szabo A G 1995 Conformational heterogeneity of tryptophan in a protein crystal J.Am.Chem.Soc. 117(8) 2321-2326

[6] Lakowicz J R 2000 On spectral relaxation in proteins Photochem.Photobiol. 72(4) 421-37

[7] Lakowicz J R Principles of fluorescence spectroscopy (Springer, New York, 2006)

[8] Pan C-P, Muino P L, Barkley M D and Callis P R 2011 Correlation of tryptophan fluorescence spectral shifts and lifetimes arising directly from heterogeneous environment J.Phys.Chem.B. 1153245 3253

[9] Weron K 1991 A probabilistic mechanism hidden behind the universal power law for dielectric relaxation - general relaxation equation J.Phys.:Condens.Matter 3 9151-9162

[10] Weron K 1992 A probabilistic mechanism hidden behind the universal power law for dielectric relaxation - general relaxation equation-replay J.Phys.:Condens.Matter 4 10507-10512

[11] Weron K and Jurlewicz A 19932 forms of self-similarity as a fundamental feature of the power-law diecectric response J.Phys.A:Math.Gen. 26 395-410

[12] Zolotarev V M , One-dimensional stable distributions (Providence, RI:American Mathematical Society, 1986)

[13] Uchaikin V V and Zolotarev V M Chance and stability. Stable distributions and their applications (VSP BV, Utrecht, 1999)

[14] Berberan-Santos M N, Bodunov E N and Valeur B 2005 Mathematical functions for the analysis of luminescence decays with underlying distributions. 1. Kohlrausch decay function (stretched exponential) Chem.Phys. 315, 171-182

[15] Berberan-Santos M N and Valeur B 2007 Luminescence decays with underlying distributions: General properties and analysis with mathematical functions J.Lumin. 126, 263-272

[16] Berberan-Santos M N, Bodunov E N and Valeur B 2005 Mathematical functions for the analysis of luminescence decays with underlying distributions. 2. Becquerel (compressed hyperbola) and related decay functions Chem.Phys. 317 57-62 
[17] Rolinski O J, Scobie K and Birch D J S 2009 Protein fluorescence decay: A gamma function description of thermally induced interconversion of amion-acid rotamers Phys.Rev.E 79 050901(R)

[18] Livesey A K, Licinio P and Delaye M 1986 Maximum entropy analysis of quasielastic light scattering from colloidal dispersions J.Chem.Phys. 84 5102-5107.

[19] Brochon J C 1994 Maximum-entropy method of data-analysis in time-resolved spectroscopy Methods Enzymol. 240 262-311.

[20] Kumar A.T.N. et al. 2001 On the rate distribution analysis of kinetic data using the maximum entropy method: Applications to myoglobin relaxation on the nanosecond and femtosecond timescales J.Phys.Chem. B, 105 7847-7856.

[21] Unruh J R, Liyanage M R and Johnson C K 2007 Tyrosyl rotamer interconversion rates and the fluorescence decays of N-acetyltyrosinamide and short tyrosyl peptides J.Phys.Chem.B. 111 5494-5502

[22] Bernardo J M and Smith A F M, Bayesian Theory (Wiley, Chichester, 1994)

[23] Cox D R and Hinkley D V, Theoretical Statistics (Chapman and Hall, London, 1974)

[24] Gelman A, Carlin J B, Stern H S and Rubin D B, Bayesian Data Analysis (Chapman and Hall, London, 1995)

[25] Gilks W, Richardson S and Spiegelhalter D Markov Chain Monte Carlo in Practice (Chapman\&Hall/CRC, 1995)

[26] Robert C P and Casella G, Monte Carlo Statistical Methods (second edition) (Springer-Verlag, New York, 2004)

[27] del Moral P, Doucet A and Jasra A 2006 Sequential Monte-Carlo samplers J. R. Statist. Soc. B 68 411-436

[28] Neal R M 2001 Annealed importance sampling, Statistics and Computing 11 125-139

[29] Burnham K P and Anderson D R, Model Selection and Multimodel Interference:A Practical Information-Theoretic Approach ( $2^{\text {nd }}$ ed.) (Springer-Verlag, ISBN 0-387-95364-7, 2002) 
Table 1. Effect of temperature, $\lambda=330 \mathrm{~nm}$.

\begin{tabular}{|c|c|c|c|c|}
\hline$T / K$ & $\tau_{0}$ & $\alpha$ & $\kappa$ & $\chi^{2}$ \\
\hline 275 & 4.988 & 0.8918 & 0.0608 & 0.997 \\
\hline 279 & 4.902 & 0.8865 & 0.052 & 1.091 \\
\hline 284 & 4.787 & 0.8813 & 0.0553 & 1.088 \\
\hline 288 & 4.609 & 0.8725 & 0.0535 & 1.042 \\
\hline 293 & 4.413 & 0.8635 & 0.0352 & 1.109 \\
\hline 299 & 4.184 & 0.9505 & 0.0043 & 1.127 \\
\hline 304 & 3.96 & 0.8444 & 0.0401 & 1.114 \\
\hline 308 & 3.753 & 0.8424 & 0.0058 & 1.043 \\
\hline 313 & 3.5864 & 0.8446 & 0.0104 & 1.043 \\
\hline 319 & 3.355 & 0.8430 & 0.0208 & 1.053 \\
\hline
\end{tabular}

Table 2. Effect of the detection wavelength $\lambda$, temperature $\mathrm{T}=294 \mathrm{~K}$.

\begin{tabular}{|c|c|c|c|c|}
\hline$\lambda / n m$ & $\tau_{0}$ & $\alpha$ & $\kappa$ & $\chi 2$ \\
\hline 325 & 4.184 & 0.8360 & 0.0049 & 1.428 \\
\hline 330 & 4.529 & 0.8632 & 0.0518 & 1.137 \\
\hline 335 & 4.7172 & 0.8769 & 0.0623 & 1.024 \\
\hline 340 & 4.872 & 0.8877 & 0.0673 & 1.029 \\
\hline 345 & 4.988 & 0.8958 & 0.0721 & 1.030 \\
\hline 350 & 5.107 & 0.9059 & 0.0814 & 1.100 \\
\hline 355 & 5.191 & 0.9110 & 0.0837 & 1.067 \\
\hline
\end{tabular}


Table 3. Comparison of AIC values obtained from fitting $I_{\alpha, k}(t)$, 2-exponential and a 3-exponential functions to the experimental data. For the 2-and 3-exponential functions, a commercial numerical procedure (DAS6 software from Horiba Jobin Yvon IBH Ltd., Glasgow, U.K.) has been used.

\begin{tabular}{|c|c|c|c|c|}
\hline $\mathbf{T} / \mathbf{K}$ & $\boldsymbol{\lambda} \mathbf{n m}$ & $\mathbf{A I C}\left(\boldsymbol{I}_{\boldsymbol{\alpha}_{\boldsymbol{K}}(\boldsymbol{t}) \mathbf{)}}\right.$ & $\mathbf{A I C}(\mathbf{2}$-exp) & AIC(3-exp) \\
\hline 275 & & 6.999 & 7.096 & 11.038 \\
279 & & 7.091 & 7.150 & 11.030 \\
284 & & 7.088 & 7.161 & 11.020 \\
288 & & 7.042 & 7.154 & 11.065 \\
293 & 330 & 7.109 & 7.219 & 11.143 \\
299 & & 7.127 & 7.230 & 11.167 \\
304 & & 7.114 & 7.260 & 11.079 \\
308 & & 7.043 & 7.187 & 11.036 \\
313 & & 7.043 & 7.205 & 11.031 \\
319 & & 7.053 & 7.229 & 11.057 \\
\hline & 325 & 7.428 & 8.403 & 12.093 \\
& 330 & 7.137 & 7.350 & 11.099 \\
294 & 335 & 7.024 & 7.119 & 10.996 \\
& 340 & 7.029 & 7.108 & 11.018 \\
& 345 & 7.030 & 7.065 & 11.009 \\
& 350 & 7.100 & 7.105 & 11.065 \\
& 355 & 7.067 & 7.085 & 11.009 \\
\hline
\end{tabular}




\section{Figure Captions:}

Fig.1. Fluorescence decay $I_{\alpha, k}(t)$ for the different parameters $\alpha$ and $\kappa(\mathbf{a})$, decay $I_{\alpha, 0}(t)$ for $\alpha=0.2,0.5,0.7$, and $1(\mathbf{b})$, and decay $I_{l, k}(t)$ for $\kappa=0,0.3,0.5$ and $0.8(\mathbf{c})$.

Fig.2. The lifetime distributions $g(\tau)$ recovered from the MEM method applied to the synthetic decay simulated for the $I_{\alpha, \kappa}(t)$ model. The parameters used are: $\tau_{0}=2 \mathrm{~ns}, \kappa=0.0001$ and $\alpha=1$, and then for the same $\tau_{0}, \kappa=0.1$ and $\alpha=1.0-0.8(\mathbf{a})$, and $\tau_{0}=2 \mathrm{~ns}, \alpha=0.9$ and $\kappa=0.05-0.3(\mathbf{b})$.

Fig.3. Example prompt function, fluorescence decay and the residuals obtained for Trp in HSA, at the temperature $\mathrm{T}=294 \mathrm{~K}$ and the detection wavelength $\lambda=335 \mathrm{~nm}$. Time correlation was 0.0141638 ns/channel. The model decay is $I_{\alpha \kappa}(t)$. The $\chi^{2}$ value for this case is 1.024.

Fig.4. The fitted parameters $\tau_{0}, \alpha$, and $\kappa$ of the fluorescence decay of $\operatorname{Tr} p$ in HSA for different detection wavelengths $\lambda$ and fixed temperature $T=294 \mathrm{~K}$ (left), and for different temperatures $T$ and fixed detection wavelength $\lambda=330 \mathrm{~nm}$ (right). The dash in the middle is the median of the estimate sample, the box corresponds to $25-75 \%$ percentiles and the whiskers show $2.5-97.5 \%$ percentiles of the estimate sample.

Fig.5. The fitted parameters $A, \Delta$, and $\sigma$ of the fluorescence decay of $\operatorname{Tr} p$ in HSA for different detection wavelengths $\lambda$ and fixed temperature $T=294 \mathrm{~K}$ (left), and at different temperatures $T$ and fixed detection wavelength $\lambda=330 \mathrm{~nm}$ (right). The dash in the middle is the median of the estimate sample, the box corresponds to $25-75 \%$ percentiles and the whiskers show $2.5-97.5 \%$ percentiles of the estimate sample.

Fig.6. Examples of model predictions compared to the experimental decays. Data shown were collected at: the wavelength of $325 \mathrm{~nm}$ and temperature $294 \mathrm{~K}$ (a), the wavelength of $355 \mathrm{~nm}$ and temperature 294 $\mathrm{K}$ (b), the wavelength of $330 \mathrm{~nm}$ and temperature $275 \mathrm{~K}$ (c), the wavelength of $330 \mathrm{~nm}$ and temperature $319 \mathrm{~K}(\mathbf{d})$.

Fig.7. $I_{\alpha, k}(t)(\mathbf{a})$ and 3-exponential (b) model predictions compared to the experimental decay collected at the wavelength of $330 \mathrm{~nm}$ and temperature $294 \mathrm{~K}$.

Fig.8. One- and two-dimensional marginal distributions of the parameter posterior for the tripleexponential model when inferred from the experimental data measured at the wavelength of $330 \mathrm{~nm}$ and temperature $294 \mathrm{~K} . \tau_{1}, \tau_{2}$, and $\tau_{3}$ are mean decay times for three exponential fluorescence components. 
$b_{1}, b_{2}$, and $b_{3}$ are corresponding scaling factors. $\Delta$ is the technical time shift parameter, and $\sigma$ is the standard deviation of the model mismatch error.

Fig.9. One- and two-dimensional marginal distributions of the parameter posterior for the $I_{\alpha, k}(t)$ model when inferred from the experimental data measured at the wavelength of $330 \mathrm{~nm}$ and temperature $294 \mathrm{~K}$. 

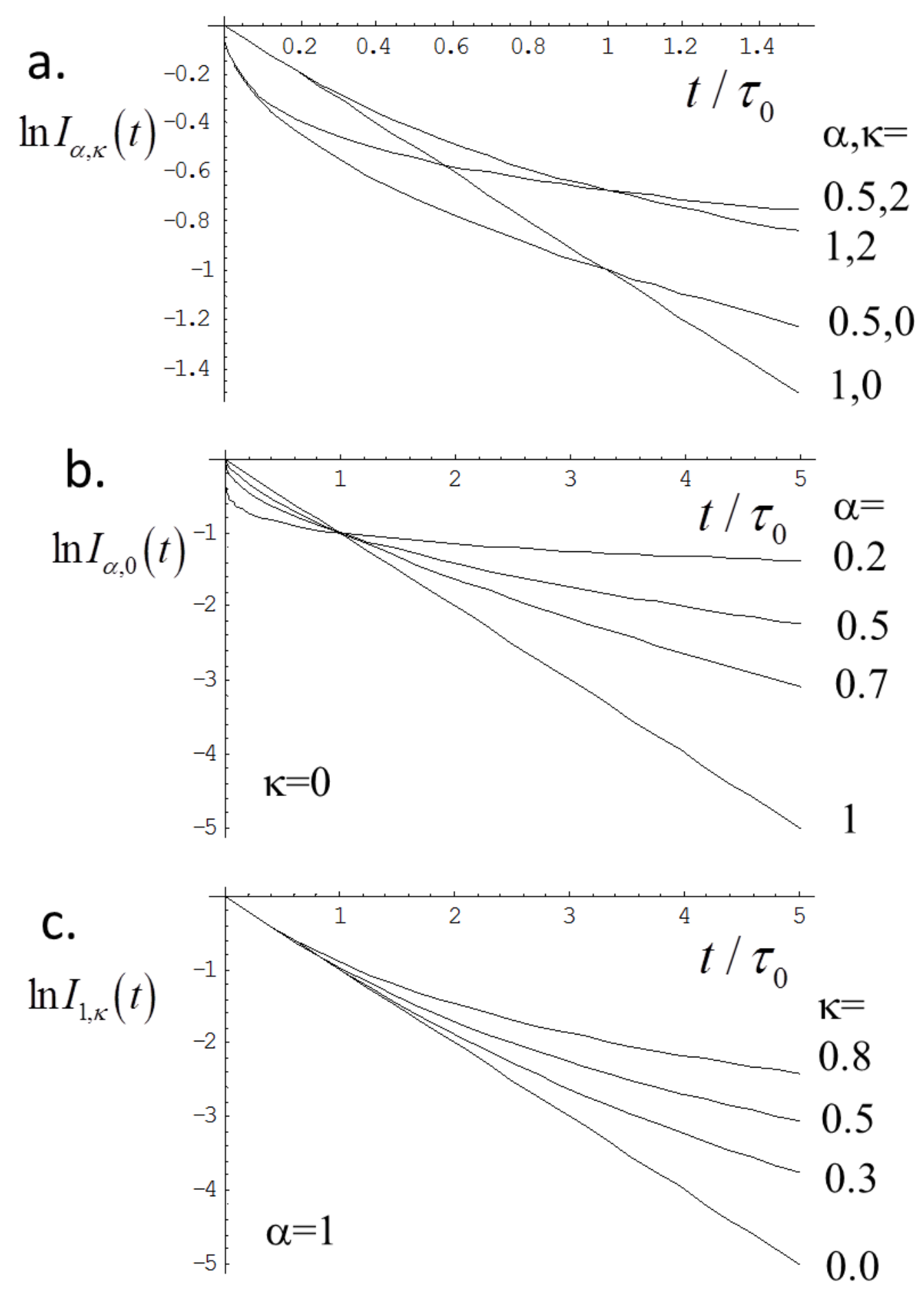

Figure 1 

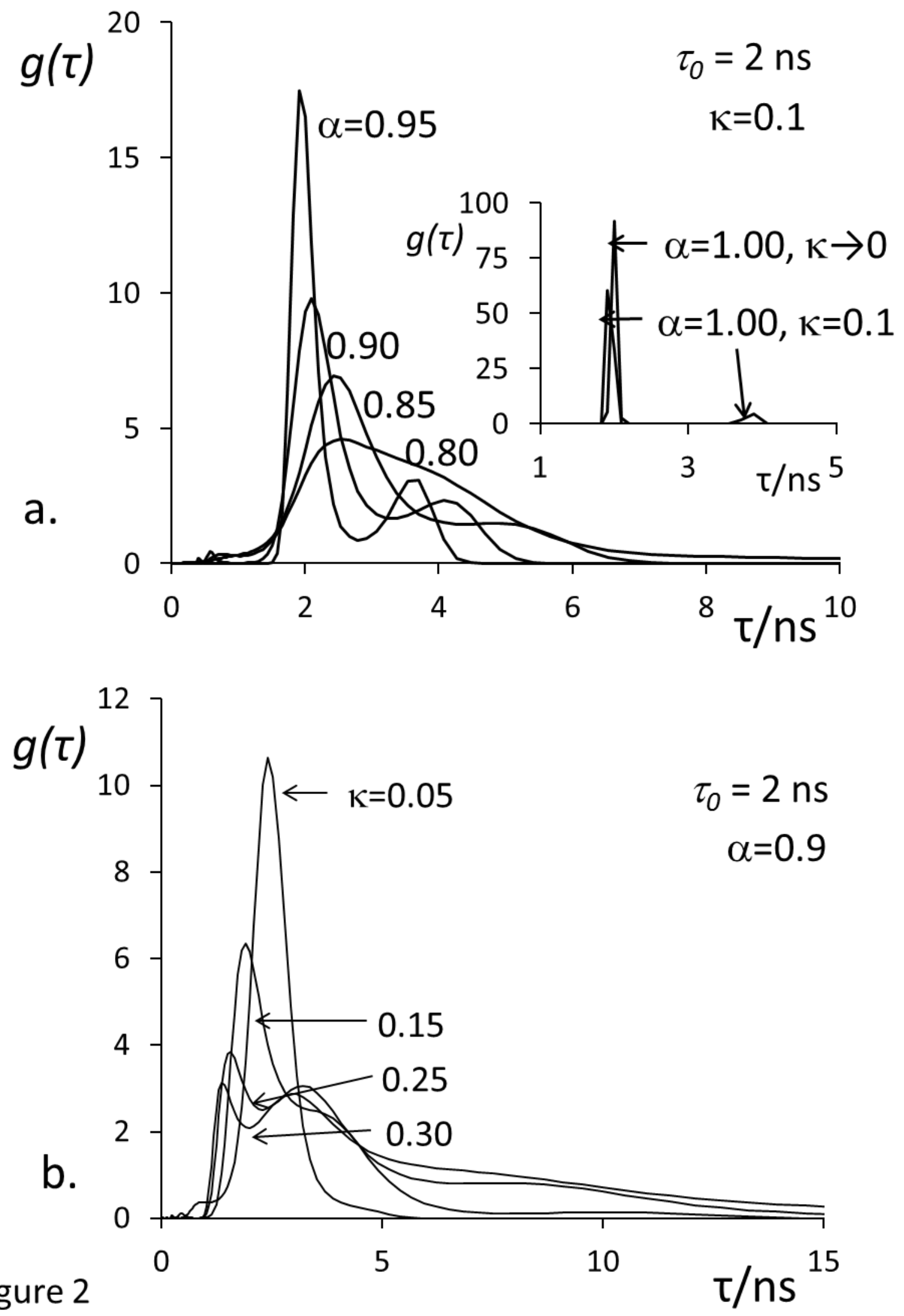

Figure 2 


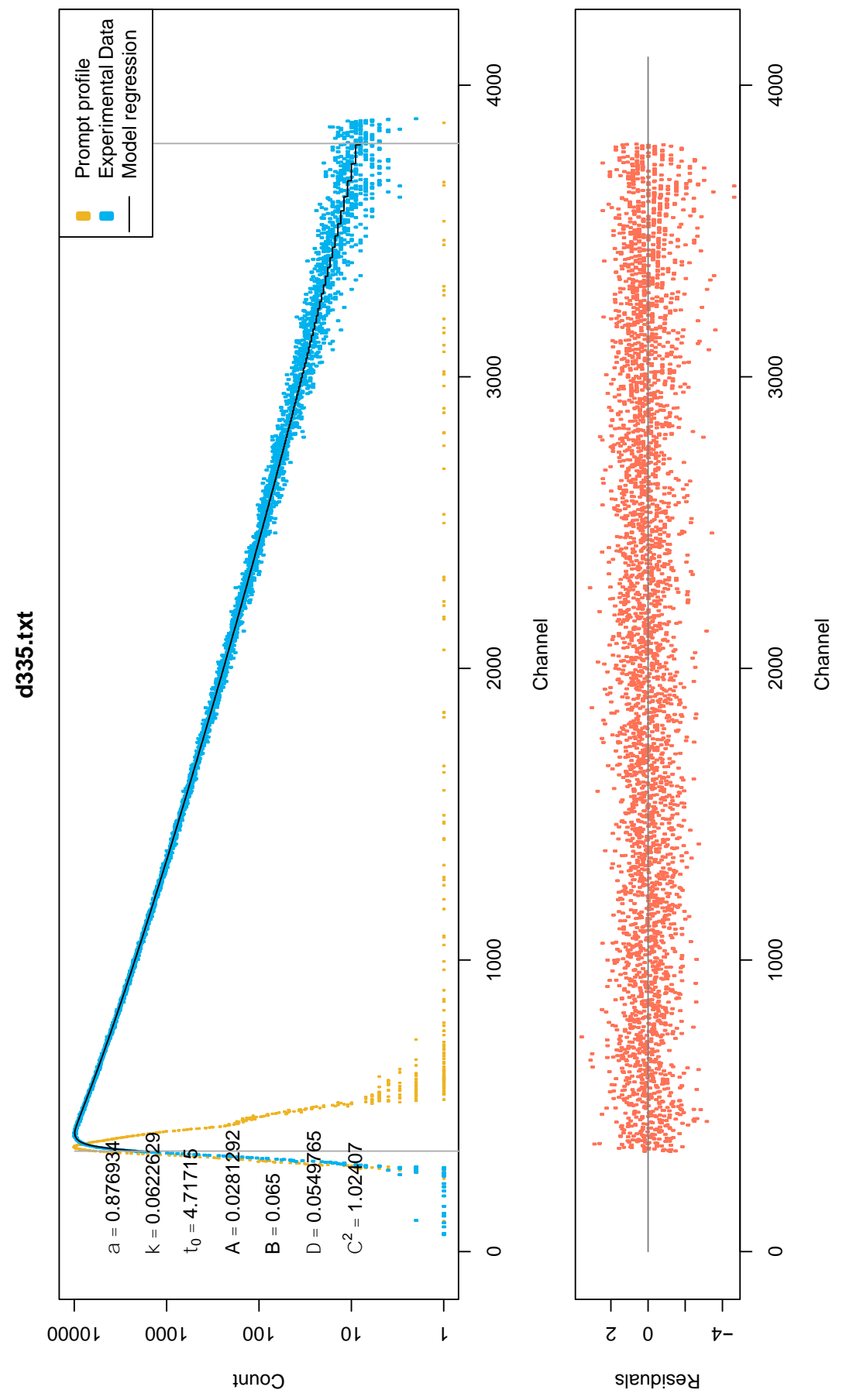

Figure 3 


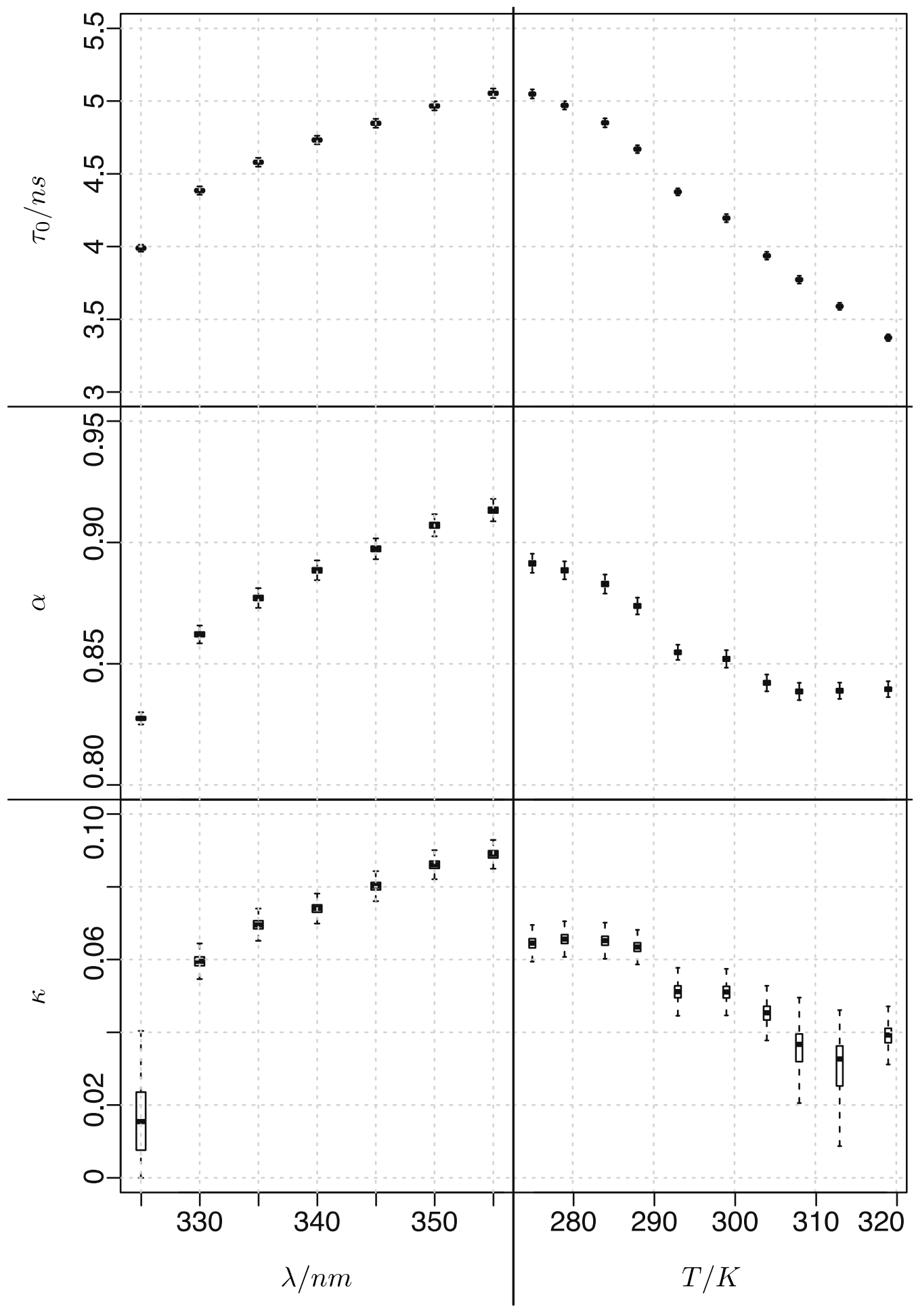

Figure 4 


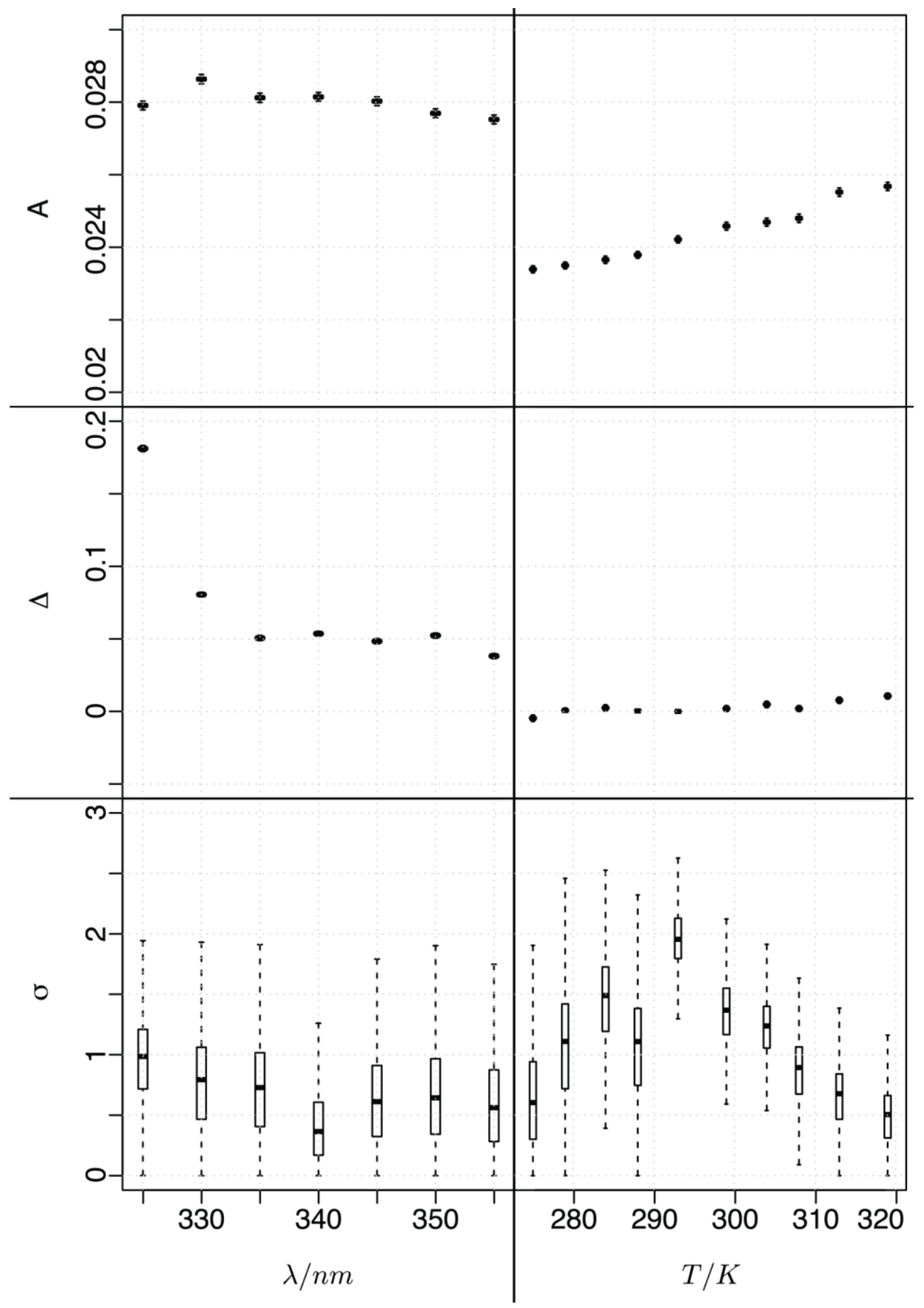

Figure 5 

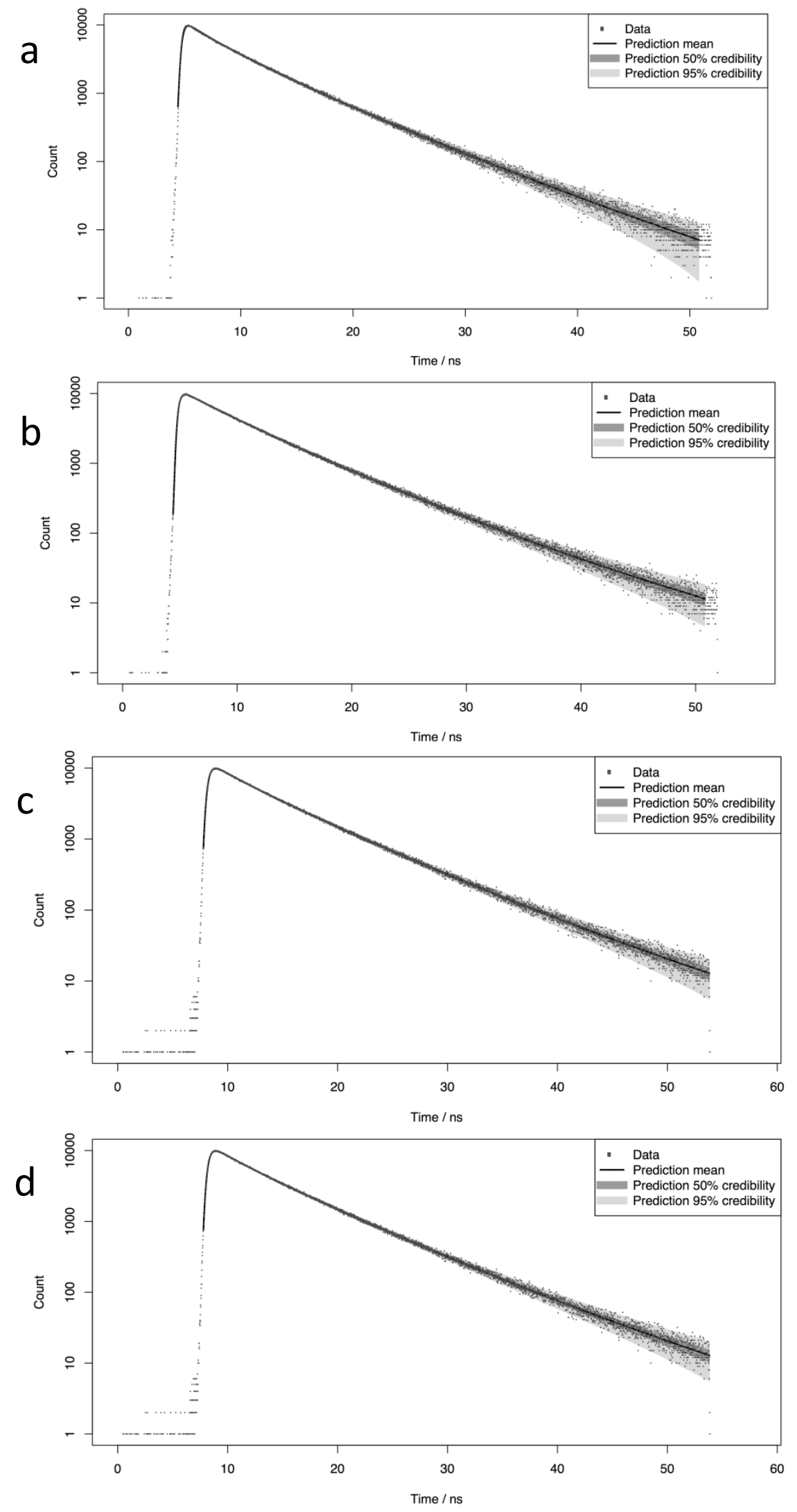

Figure 6 

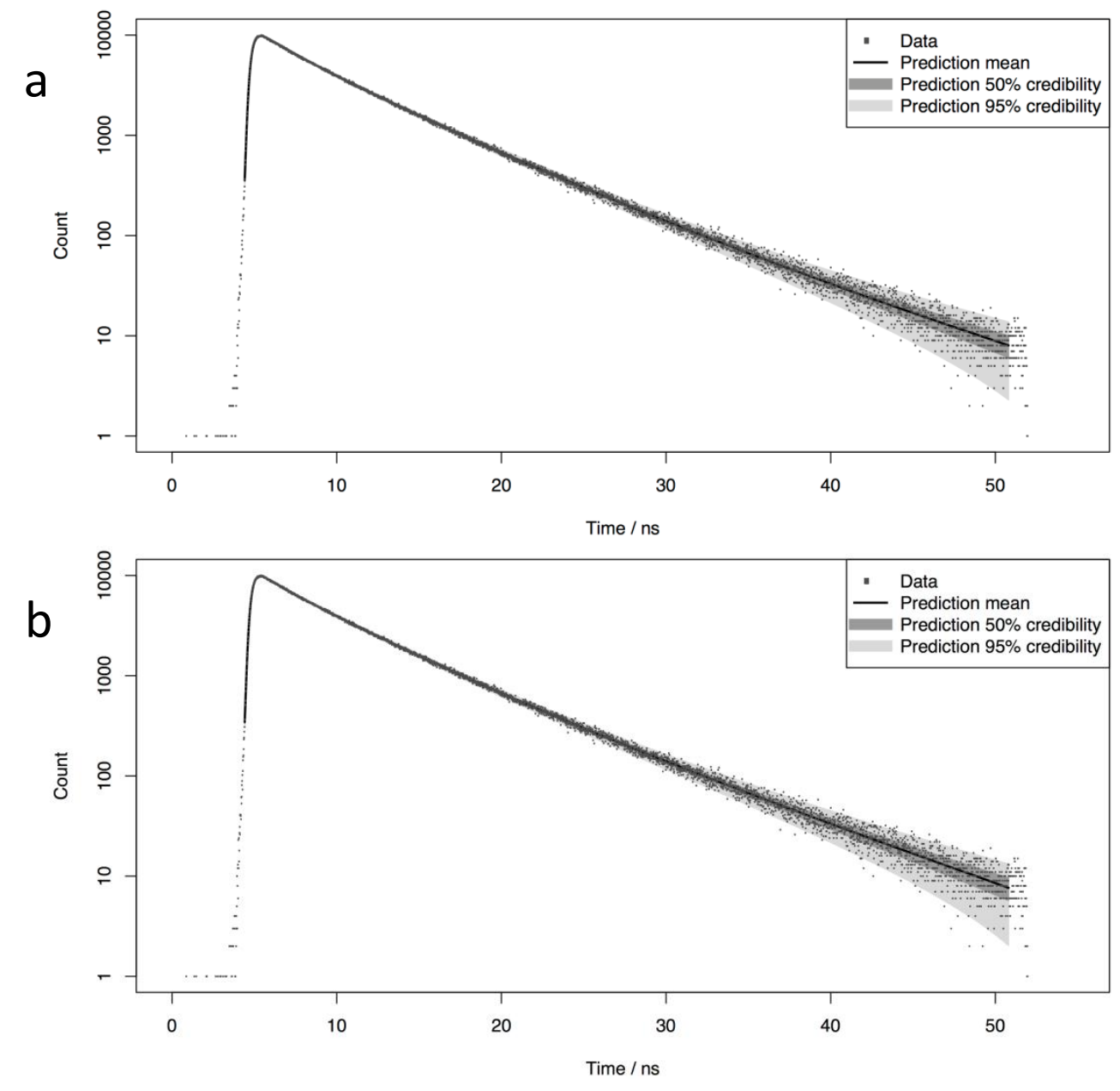

Figure 7 


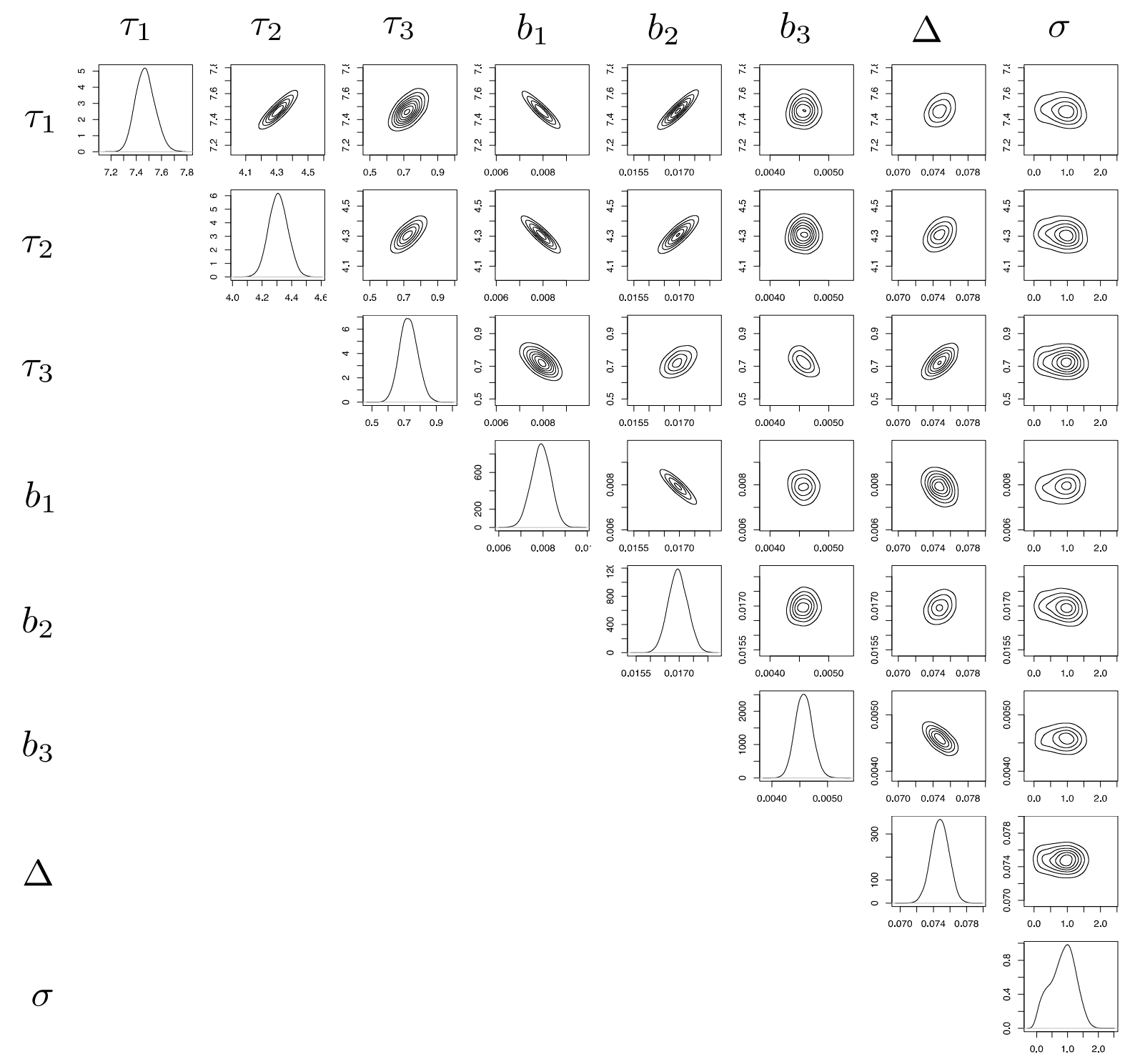

Figure 8 

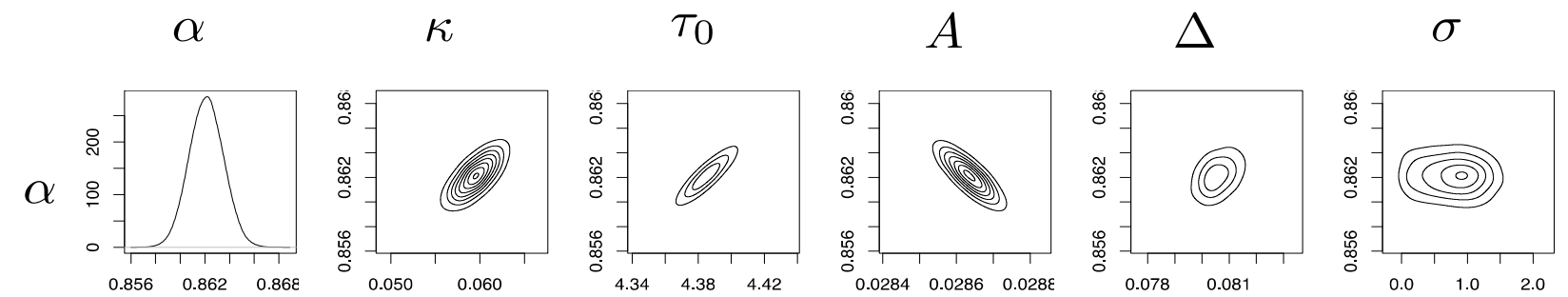

$\kappa$
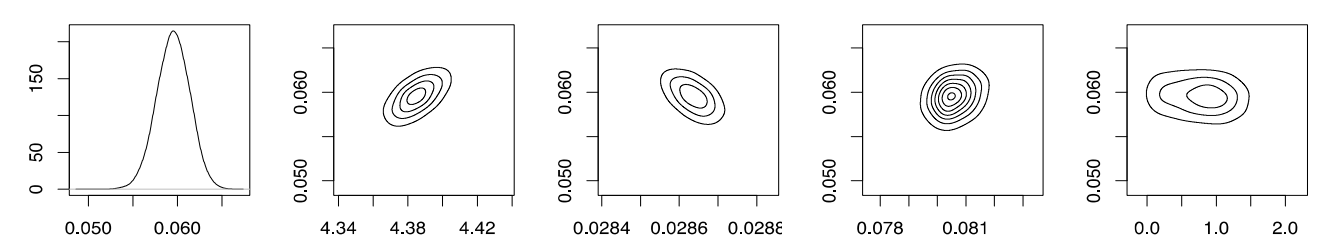

$\tau_{0}$
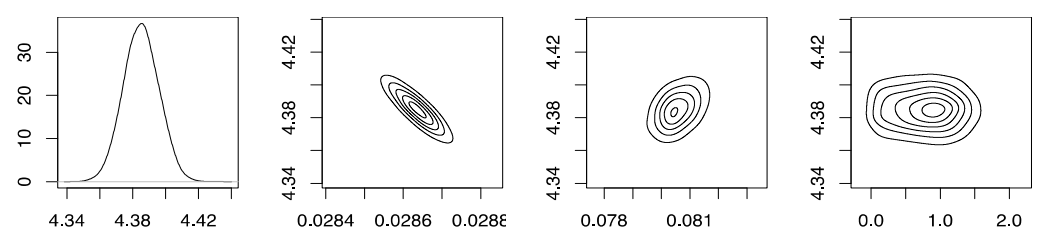

A
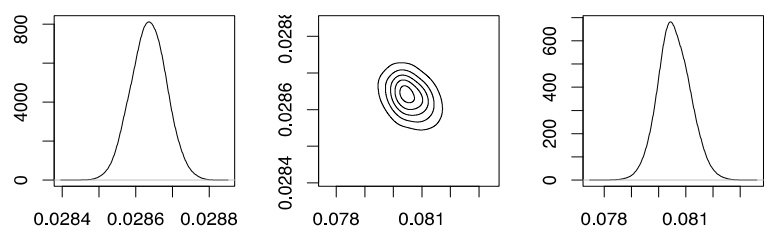

$\Delta$
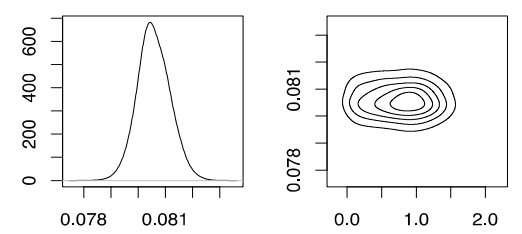

$\sigma$

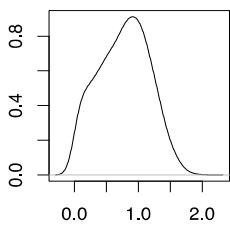

Figure 9 